\title{
Same Donor Laparoscopic Liver and Kidney Procurement for Sequential Living Donor Liver-Kidney Transplantation in Primary Hyperoxaluria Type I
}

\author{
Roberta Angelico, MD, PhD, FEBS, Isabella Guzzo, MD, ${ }^{1}$ Marco Pellicciaro, MD, \\ Maria Cristina Saffioti, MD, ${ }^{1}$ Chiara Grimaldi, MD, ${ }^{1}$ Chebl Mourani, MD, ${ }^{3}$ Francesco Smedile, MD, ${ }^{4}$ \\ Rosanna Pariante, MD, ${ }^{4}$ Alessia Semprini, MD, ${ }^{5}$ Lidia Monti, MD, ${ }^{5}$ \\ Manila Candusso, MD, ${ }^{6}$ Luca Dello Strologo, MD, ${ }^{2}$ and Marco Spada, MD, PhD, FEBS ${ }^{1}$
}

\begin{abstract}
Background: Sequential liver-kidney transplantation (SeqLKT) from the same living donor has shown excellent results in children with primary hyperoxaluria type 1 (PH1), yet its experience is limited due to the invasiveness of two major procedures for liver-kidney procurement in a single donor. Despite laparoscopic nephrectomy and hepatic left lateral sectionectomy (LLS) being considered standard procedures in living donation, the sequential use of the two laparoscopic approaches in the same living donor has never been reported.

Methods: Herein, we present the first two case series of laparoscopic liver-kidney procurement in the same living donor for SeqLKT in children with PH1 and review of the current literature on this topic.

Results: In the first case, a 15-month-old boy received a SeqLKT from his 32-year-old mother, who underwent a laparoscopic LLS and, after 8 months, a laparoscopic left nephrectomy. In the second case, a 34-month-old boy received a SeqLKT from his 40-year-old father who underwent laparoscopic LLS followed by handassisted right nephrectomy after 4 months. Both donors had uneventful postoperative courses and were discharged within 5 days from each surgery. The first recipient had no complication; the second child after liver transplantation developed a partial thrombosis of the inferior vena cava, which did not preclude the sequential kidney transplantation. After 12 months, donors and recipients displayed normal liver and renal functions.

Conclusions: Sequential laparoscopic liver-kidney procurement in the same living donor is safe and feasible, and might be considered as a possible strategy to promote SeqLKT in children with PH1 from the same living donor.

Keywords: living donation, laparoscopic liver and kidney procurement, primary hyperoxaluria, liver transplantation, kidney transplantation, mini-invasive surgery

Introduction

$\mathbf{P}$ RIMARY HYPEROXALURIA TYPE 1 (PH1) is a rare autosomal recessive metabolic disorder caused by a deficiency of the liver enzyme alanine-glyoxylate aminotransferase, resulting in increased hepatic oxalate production. The excess of oxalate causes progressive end-stage renal disease (ESRD) and systemic oxalosis, leading to severe morbidity and mortality. ${ }^{1}$

Combined liver-kidney transplantation (CLKT) is considered the treatment of choice in patients affected by PH1 with ESRD. ${ }^{1}$

Liver transplantation (LT) may be simultaneous or sequential with kidney transplantation (KT) depending on disease staging, renal function, and type of donor availability. ${ }^{2}$ Initially, simultaneous or sequential CLKT has been mainly performed using organs from deceased donors. ${ }^{3}$ Because of organ shortage, sequential liver-kidney transplantation (SeqLKT) from the same living donor has been proposed in PH1 patients, showing biochemical and immunological advantages for the recipient. ${ }^{4}$ Yet, the experience of SeqLKT from the same living donor is limited due to the complexity of the donor surgical procedures. ${ }^{5-12}$
\end{abstract}

\footnotetext{
${ }^{1}$ Division of Abdominal Transplantation and Hepatobiliopancreatic Surgery, Bambino Gesù Children's Hospital IRCCS, Rome, Italy.

${ }^{2}$ Department of Nephrology and Dyalisis, Bambino Gesù Children's Hospital IRCCS, Rome, Italy.

${ }^{3}$ Department of Pediatrics, Hôtel-Dieu de France Hospital (HDF), Beirut, Lebanon.

${ }^{4}$ Department of Anesthesiology, Bambino Gesù Children's Hospital IRCCS, Rome, Italy.

${ }^{5}$ Department of Radiology, Bambino Gesù Children's Hospital IRCCS, Rome, Italy.

${ }^{6}$ Division of Hepatology and Gastroenterology, Bambino Gesù Children's Hospital IRCCS, Rome, Italy.
} 
Although in living donation the laparoscopic left lateral sectionectomy (LLS) ${ }^{13,14}$ and nephrectomy ${ }^{15,16}$ showed good donor and recipient outcomes, the laparoscopic sequential liver-kidney procurement has never been reported.

To the best of our knowledge, we report the first two cases of sequential laparoscopic liver-kidney procurement from the same living donor for SeqLKT in children with PH1. The literature of living-related SeqLKT for $\mathrm{PH} 1$ patients has been systematically reviewed.

\section{Center Experience}

\section{Case 1}

A 15-month-old boy (weight: $8 \mathrm{~kg}$; height: $74 \mathrm{~cm}$ ) with genetically confirmed PH1 and ESRD was referred for CLKT. At presentation, the patient was on peritoneal dialysis with secondary hyperparathyroidism, hyperphosphatemia, and anemia. Serum oxalates were $128 \mu \mathrm{mol} / \mathrm{L}$ and hemodialysis was started (in addition to nocturnal peritoneal dialysis) to reduce his level. The family was offered the option of a living-related SeqLKT, and the 32-year-old mother (weight: $67 \mathrm{~kg}$; body mass index [BMI]: 23.5) expressed her willingness to donate. Her evaluation revealed no physical or psychological contraindications for living donation. Computed tomography (CT) and three-dimensional reconstruction were used to define the donor anatomy and the expected liver graft volume (Fig. 1). Liver vascular anatomy consisted in an aberrant left hepatic artery (HA) arising from the left gastric artery and a right HA from the proper HA, while the left portal vein (PV), the hepatic veins (HVs), and biliary tree had standard anatomy. The expected left lateral segment (SIISIII) volume was 243 gr (graft-to-recipient weight ratio
[GRWR]: 3\%). At the CT, the left kidney had conventional anatomy (one renal artery, one renal vein, one ureter) and was chosen for SeqLKT.

First, in the donor full laparoscopic LLS was carried out as described by Soubrane et al. ${ }^{17}$ The patient was placed in supine position, with her legs apart to apply the French position. The pneumoperitoneum was established at $12 \mathrm{mmHg}$ and five trocars were inserted (Fig. 2A). Within the lesser omentum, the left HA was isolated. The round ligament was dissected and all the branches for segment IV were divided, exposing the left PV. After the sectioning of the Arantius ligament, the left HV was encircled extra-parenchymally. To obtain a modified "hanging maneuver," an umbilical tape was placed between the left $\mathrm{HV}$ and the middle $\mathrm{HV}$, and passed over the left PV and left HA. Dissection of the parenchyma was then performed to the right of the falciform ligament, using bipolar forceps, clips, sutures, and sealing devices, without Pringle maneuver. To preserve vascularization of the left bile duct, the hilar plate was sharply divided at the level of the Rex recessus, and the stump of the bile duct of the donor's remnant liver was closed with sutures. When the parenchymal transection was completed, the left HA, the left PV, and the left HV were sequentially divided using mechanical staplers. A Pfannenstiel incision was used for graft extraction, and after harvesting the graft was flushed with preservation solution (Celsior) on the back table. During donor's surgery, blood transfusion was not required. The donor had an uneventful postoperative course and was discharged after 5 days.

In the recipient, due to elevated preoperative oxalate levels a bilateral nephrectomy was performed. After hepatectomy with inferior vena cava (IVC) preservation, the left lateral segment was transplanted using the piggy-back technique. ${ }^{18}$
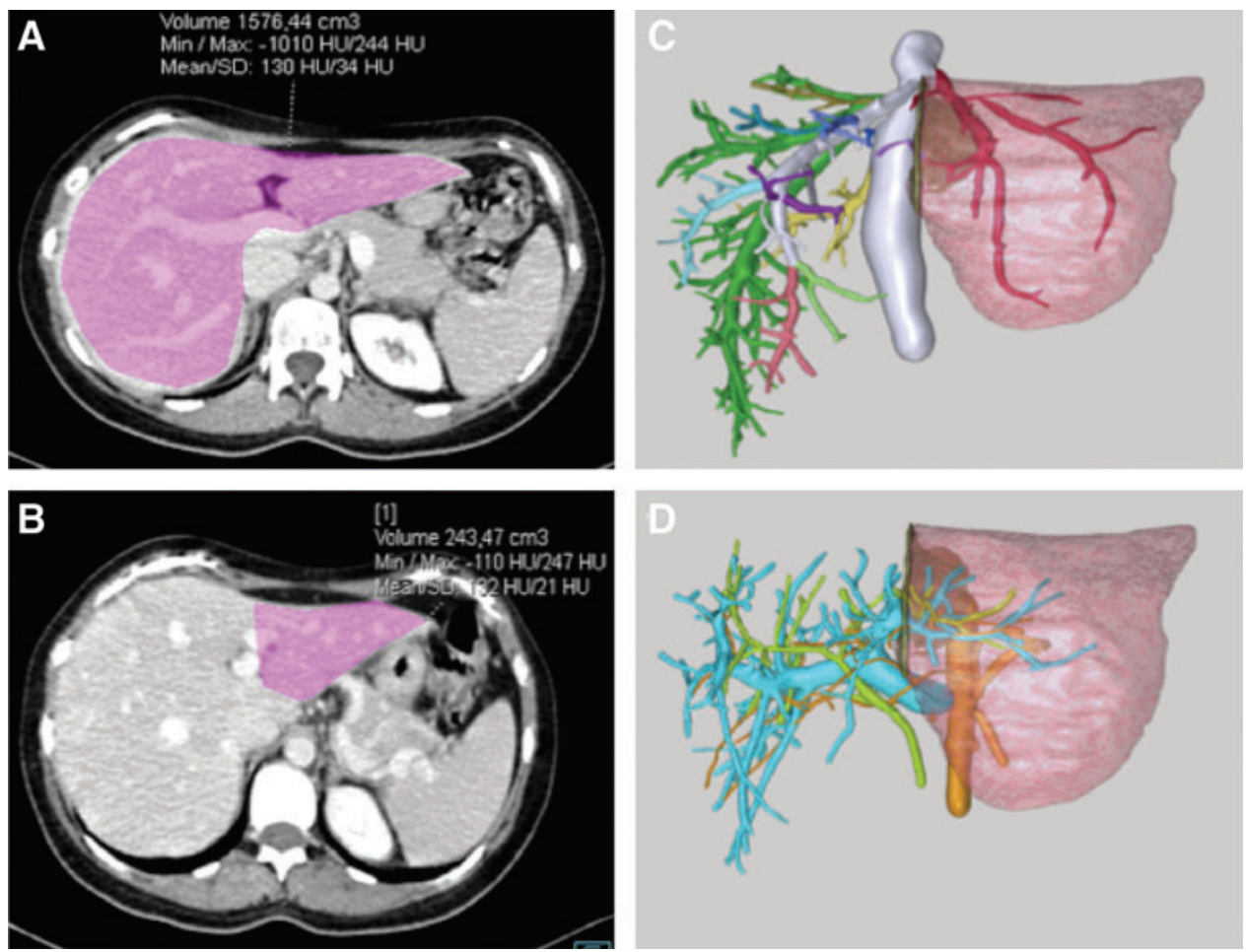

FIG. 1. Preoperative donor imaging of the liver. Preoperative donor's CT scan showing the volumetry of (A) the whole liver and (B) the left lateral segment; three-dimensional reconstruction of (C) the hepatic vein outflow and (D) the portal vein, hepatic artery, and bile duct anatomy. CT, computed tomography. 


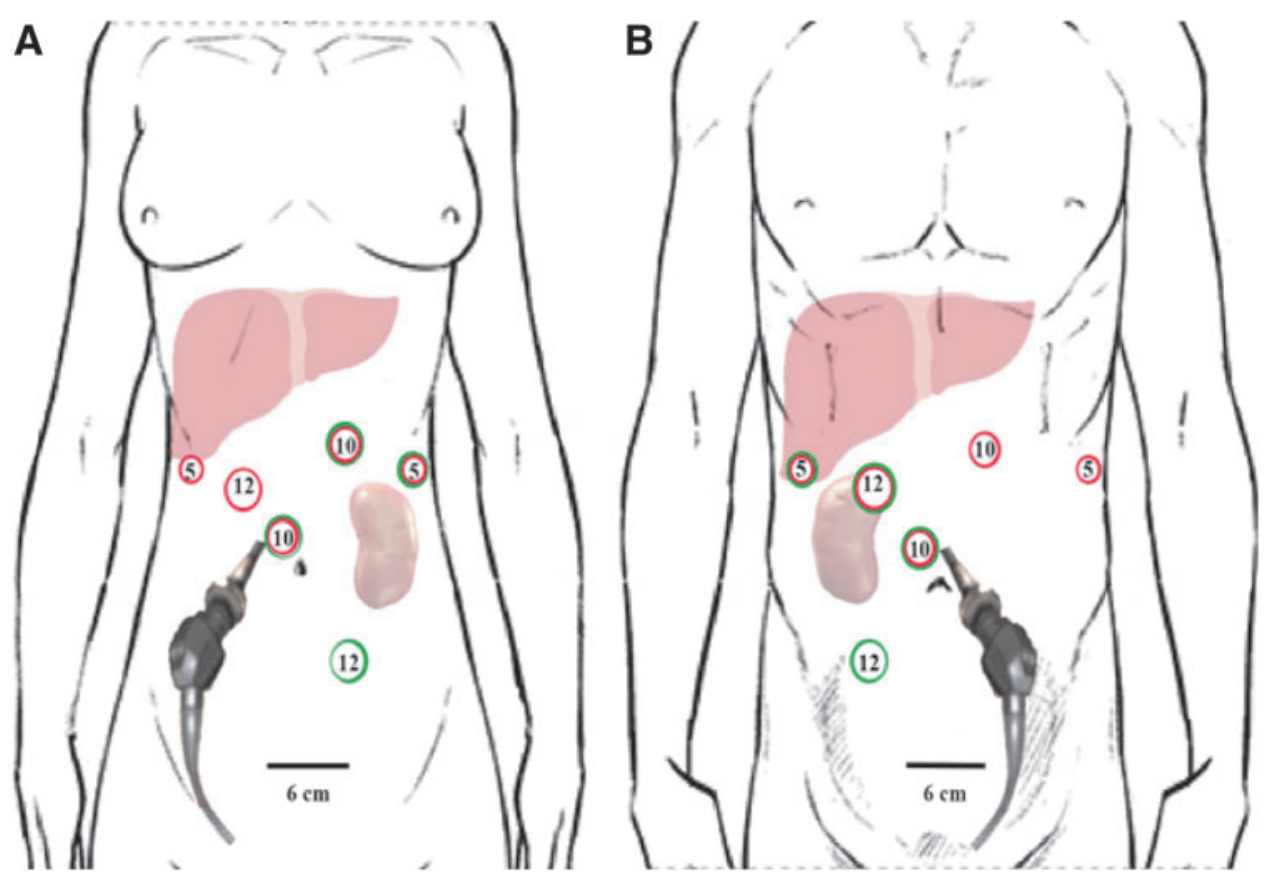

FIG. 2. Trocar position for sequential laparoscopic left lateral sectionectomy and nephrectomy in the same living donor. (A) Trocar position for laparoscopic left lateral sectionectomy (red) and left nephrectomy (green). (B) Trocar position for laparoscopic left lateral sectionectomy (red) and right-hand-assisted nephrectomy (green). For both sequential laparoscopic nephrectomies, two $10 \mathrm{~mm}$-trocar and one 5-mm trocar incisions performed for the liver surgery were reused. The same Pfannenstiel incision was used for both liver and kidney extractions.

After LT, liver function tests gradually normalized and hemodialysis was continued (six times/week). Recipient's postoperative course was characterized by chronic thrombocytopenia and anemia, and two spontaneous bone fractures, which required a delay of KT until complete recovery.

After 8 months, the oxalate plasma level decreased to $50 \mu \mathrm{mol} / \mathrm{L}$, and the living-related KT was carried out. In the donor, four trocars were inserted [three trocars using the same incision previously performed for LLS, one additional 12-mm trocar placed in the inferior left iliac fossa (Fig. 2A)]. At abdominal exploration, no adhesions were found, and a full laparoscopic left nephrectomy was performed as described by Ratner et al. ${ }^{19}$ The pre-existing Pfannenstiel incision was used for kidney extraction. The mother had an uneventful postoperative course and was discharged after 5 days. In the recipient, KT was performed without complications. Hemodialysis was continued for the first 10 days postsurgery to ensure complete removal of oxalate and stopped thereafter. After 12 months, the recipient had normal liver and renal functions (Fig. 3); the donor is currently in good condition with normal biochemical tests (creatinine: $0.8 \mathrm{mg} / \mathrm{dL}$; aspartate aminotransferase [AST]: $23 \mathrm{IU} / \mathrm{L}$; total bilirubin: $0.4 \mathrm{mg} / \mathrm{dL}$ ).

\section{Case 2}

A 34-month-old boy (weight: $11 \mathrm{~kg}$; height: $86 \mathrm{~cm}$ ) affected by genetically confirmed PH1 and ESRD, on peritoneal dialysis since the age of 2 months, was referred for CLKT. During the pretransplant investigation, the CT showed a thrombosis of the right iliac vein, but a patent left iliac vein. Oxalate plasma level was $160 \mu \mathrm{mol} / \mathrm{L}$, and hemodialysis was started to maximize its removal. The 40-yearold father (weight: $67 \mathrm{~kg}$; BMI: 24) proposed himself as a candidate donor and, after multidisciplinary evaluation, he was defined physically and psychologically suitable to donate. The father's CT showed a conventional liver anatomy and an estimated left lateral segment of 328 gr (GRWR: $2.9 \%$ ); three renal arteries and one renal vein were detected in the left kidney, while single renal artery and vein in the right kidney, which was chosen for SeqLKT.

The donor liver surgery consisted in laparoscopic LLS without Pringle maneuver and blood transfusion requirement as described in Case 1 (to identify the left HA arising from the proper HA, the left side of the hepatic pedicle was dissected). Trocar positions are detailed in Figure 2B. The donor had an uneventful postoperative course and was discharged 5 days after surgery. In the recipient, after bilateral nephrectomy the left lateral segment was transplanted as defined in Case 1. After $\mathrm{LT}$, a rapid normalization of liver function tests was observed, while hemodialysis was continued six times/week. Two months from surgery the child developed ascites. The angioCT and cavography showed normal suprahepatic outflow, but thrombosis of the retrohepatic IVC; the left iliac vein and subhepatic IVC outflows were passing through the lumbar veins into the azigos vein (Fig. 4). Anticoagulants and diuretics were started, with a progressive resolution of the ascites.

After 4 months from LT, serum oxalate level was $49 \mu \mathrm{mol} / \mathrm{L}$ and KT was planned. The father underwent a right-handassisted nephrectomy with standard technique, ${ }^{20}$ for which three trocar incisions previously performed for the LLS were used, and one 12-mm trocar incision was added in the inferior right iliac fossa (Fig. 2B). The pre-existing Pfannenstiel incision was used for the hand insertion and graft extraction. The donor postnephrectomy course was uneventful, and the patient was discharged after 4 days. In the recipient, KT was successfully performed in the left iliac fossa, and stable 

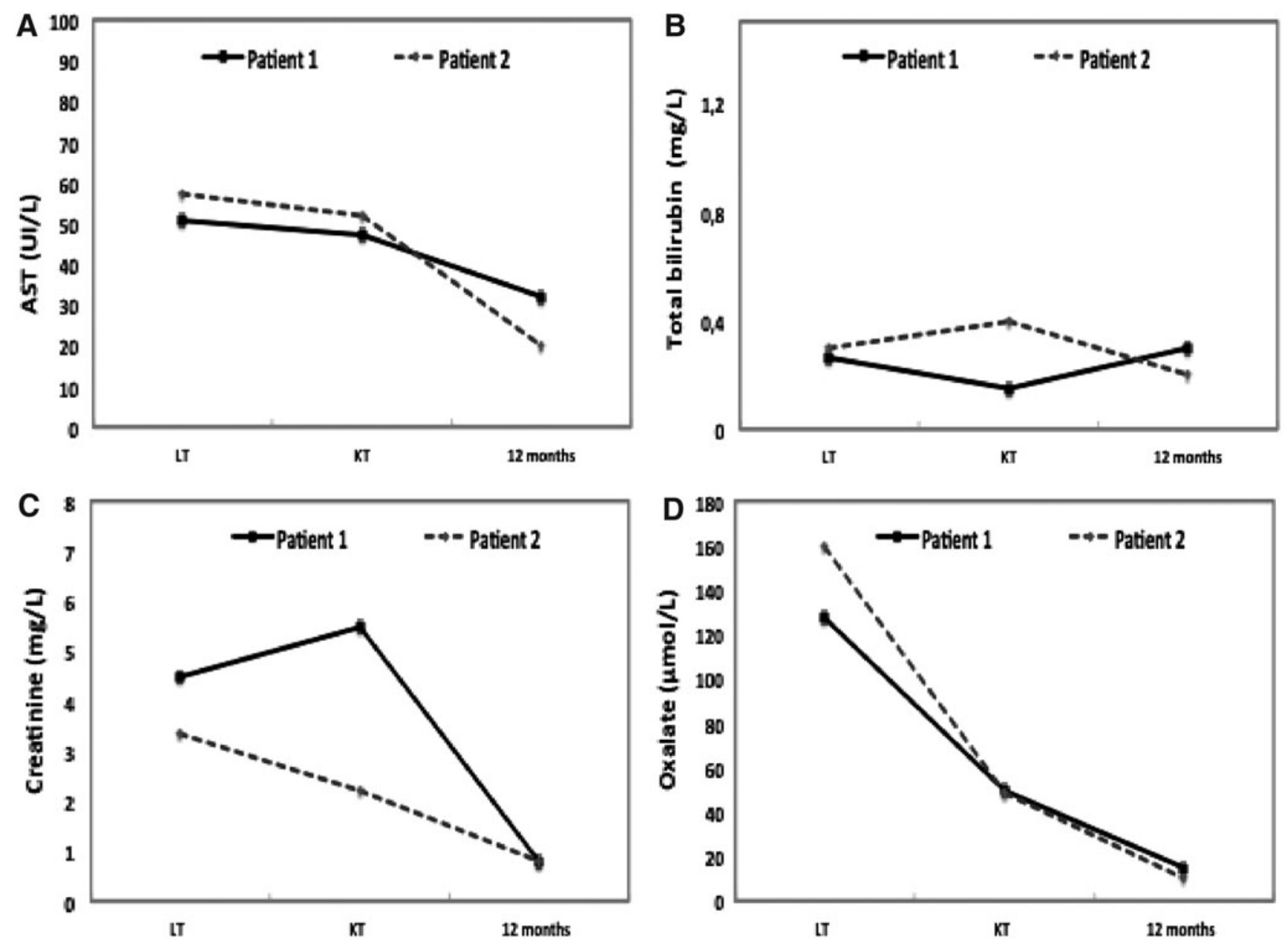

FIG. 3. Recipients' liver-kidney functions (A-C) and plasma oxalate levels (D). AST, aspartate aminotransferase; KT, kidney transplantation; LT, liver transplantation.

liver and renal functions were maintained throughout the entire follow-up (Fig. 3). After 12 months from surgery, the donor is in good clinical condition, with normal biochemical tests (creatinine: $1.1 \mathrm{mg} / \mathrm{dL}$; AST: $35 \mathrm{U} / \mathrm{L}$; total bilirubin: $0.7 \mathrm{mg} / \mathrm{dL})$.
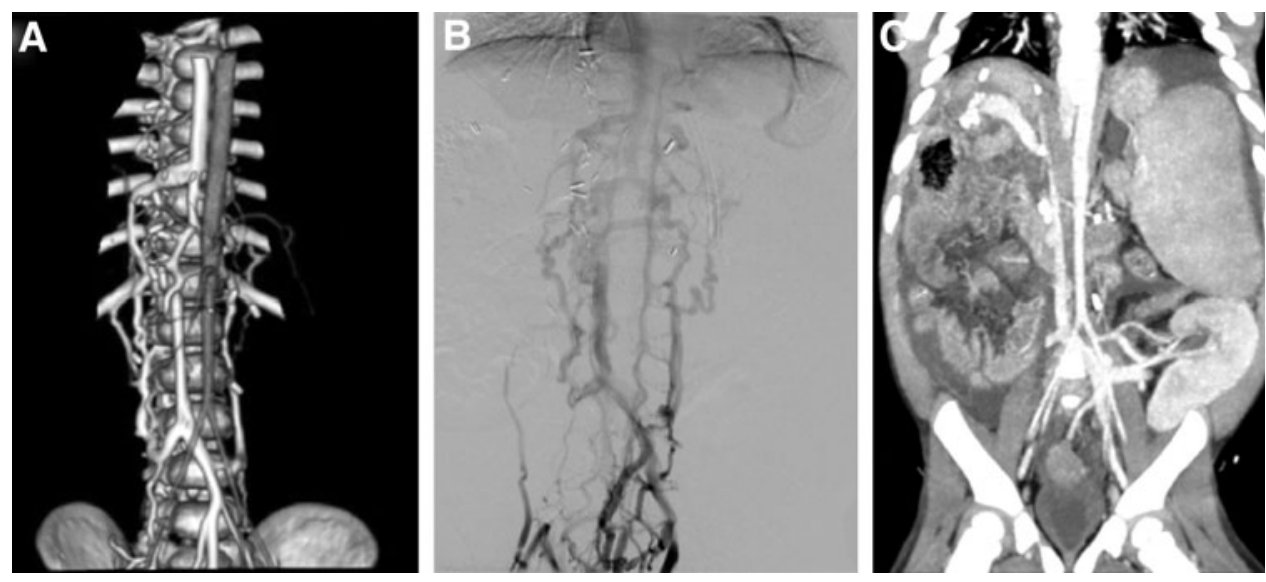

FIG. 4. Case 2 angio-CT scan and cavography. Case 2 recipient's (A) angio-CT scan and (B) cavography, after 2 months from liver transplantation, showing the thrombosis of the inferior vena cava and right iliac vein but patent left ilac vein and good outflow of the lower extremities through the retroperitoneal vein system; (C) angio-CT scan after kidney transplantation in the left iliac fossa. 
systemic oxalosis is present, SeqLKT (first LT, then dialysis until sufficient oxalate clearance from the body, followed by KT) seems to be preferable compared with simultaneous CLKT. ${ }^{1,22}$ However, the timing for KT is influenced by the recipient's oxalate pool as well as the deceased donor kidney availability. ${ }^{1}$

In the current era of organ shortage, the living-related SeqLKT in PH1 recipients combines the advantages of a sequential procedure-which optimizes the renal function by performing the KT when stable LT function and low plasma oxalate levels are achieved - and the benefits of a livingrelated transplantation, reducing the KT waiting-list time and the dialysis-related complications.

So far, SeqLKT from the same living donor in PH1 recipients has been reported only in small series (Table 1). ${ }^{5-12}$ Of 23 cases described in the literature, the median recipients' age at SeqLKT was $6(1-41)$ years and the majority of recipients $(74 \%)$ received a left lateral segment graft. Mor et al. reported that SeqLKT from the same living donor could be safely performed also in young adults with PH1 using a right liver lobe graft. ${ }^{9}$ The interval between LT and KT was $\sim 6$ (1-10.6) months, during which period dialysis was performed according to center's protocol (usually two to three times/week). Postoperative recipients' complications included bleeding $(n=2)$, cytomegalovirus infection $(n=3)$ and bile leak $(n=1)$ and biliary sepsis $(n=1)$. After a follow-up of $2.3(0.2-19.7)$ years, the overall recipient patient survival was $88.5 \%$, while liver and kidney graft survivals were $88.5 \%$ and 90.5\% (two KT lost for rejection), respectively, which appears superior to those reported in pediatric CLKT from deceased donors (2-year survivals: $72.4 \%$ patient survival; $60.3 \%$ liver graft survival; $55.2 \%$ renal graft survival). ${ }^{21}$

On the donor side, no mortality and morbidity were reported after sequential liver-kidney procurements in the same living donor, except for one patient who developed an abdominal fluid collection after liver resection, resolved by percutaneous drainage. All donors had a rapid postoperative recovery, and showed normal liver and renal function tests in the long term (range: 2.7 months-19.7 years). ${ }^{5-12}$

Despite these promising results, the number of SeqLKT from the same living donor is limited, most likely because of the invasiveness of the two donor surgeries using the open technique and the lack of guidelines recommending this procedure.

In the last two decades, the laparoscopic hand-assisted or pure laparoscopic nephrectomies have been established as the gold standard, being preferred to the open procedure due to less postoperative pain, shorter hospital stay, and earlier return to normal activities. $15,16,23,24$

Also for living liver donation, a laparoscopic approach to partial hepatectomy showed advantages over the open technique in terms of intraoperative blood losses, postoperative recovery, and abdominal wall complications. ${ }^{13,14}$ According to recent consensus guidelines, donor laparoscopic LLS for adult-to-child LT may be regarded as standard procedures, but it is still limited to few highly specialized centers. ${ }^{25,26}$ Recently, the feasibility of the combination of the two laparoscopic techniques in the same living donor was described by Gautier et al. for simultaneous CLKT. ${ }^{27}$

Of 227 pediatric LTs performed at our center between September 2008 and December 2018, 12 (5.3\%) children underwent CLKT for PH1 (five simultaneous CLKT [one from living donor], seven SeqLKT [four from living donor]). Following a 15-year learning curve in laparoscopic living donation, we adopted the laparoscopic approach for both liver and kidney procurements in the last two cases, which represent the first series of sequential laparoscopic LLS and nephrectomy in the same living donor.

In our experience, the sequential laparoscopic liver-kidney procurement in the same donor was safe and feasible, providing several advantages: on the donor side, it combined the benefits of the two laparoscopic procedures (when performed separately) maintaining the surgical risks independent for each operation; on the recipient side, the sequential procedures permitted to optimize the KT timing according to recipients' needs. Moreover, the donor LLS was associated with a rapid postoperative recovery without significant psychological/physical trauma (which would reduce the will to undergo a second operation) and permitted to reuse the same surgical incisions for the kidney procurement offering good esthetic results.

In the two PH1 recipients, liver and kidney functions rapidly recovered after transplantation, providing evidence that the laparoscopic donor procedures had no impact on graft outcomes. In the second case, the recipient developed IVC thrombosis after LT, which could be related to the pre-existing right iliac vein thrombosis and/or to the bilateral native nephrectomies performed after the hepatectomy. As proposed in other series, ${ }^{28,29}$ in our recipients the bilateral nephrectomy at the time of LT was performed to remove the significant stores of oxalate contained in the native kidneys, which may cause damage to the newly transplanted renal graft.

In our experience, the living donor laparoscopic sequential liver-kidney procurement might be proposed in well-selected cases, requiring (i) a robust indication for SeqLKT in PH1 recipients (according to clinical status, age, and oxalate levels); (ii) meticulous living-related donor assessment (age $\leq 55$ years, BMI $<30$, absence of active infections/malignancies/ systemic diseases, glomerular filtration rate $\geq 70-80 \mathrm{~mL} / \mathrm{min} /$ $1.73 \mathrm{~m}^{2}$, definition of liver/kidney anatomy and liver volume, psychological evaluation); and (iii) adequate donor-recipient matching (ABO compatibility, liver GRWR between 1.5 and 3). Yet, the laparoscopic living donor approach for SeqLKT has limitations related to the surgical technical complexity, which requires sufficient learning curve in mini-invasive liver-kidney procurement, and to the exposure of the same living donor to two major surgeries. So far, donor hepatectomy has imposed $0.1 \%-0.2 \%$ mortality and $25 \%-35 \%$ morbidity (mainly biliary leak) on healthy individuals, ${ }^{30}$ while donor nephrectomy has shown $<0.03 \%$ mortality and $10 \%-20 \%$ morbidity (major complication $<3 \%) .{ }^{31}$ Therefore, the potential living donor for SeqLKT has to be adequately informed of the risks for each surgical procedure.

Although additional experiences are needed, the two cases described herein suggest that sequential laparoscopic LLS and nephrectomy in the same living donor are safe and feasible, and could be considered as a preferential strategy to promote living-related SeqLKT in children affected by PH1.

\section{Patient Consent}

Informed consent was obtained from the patient for publication of their information. 


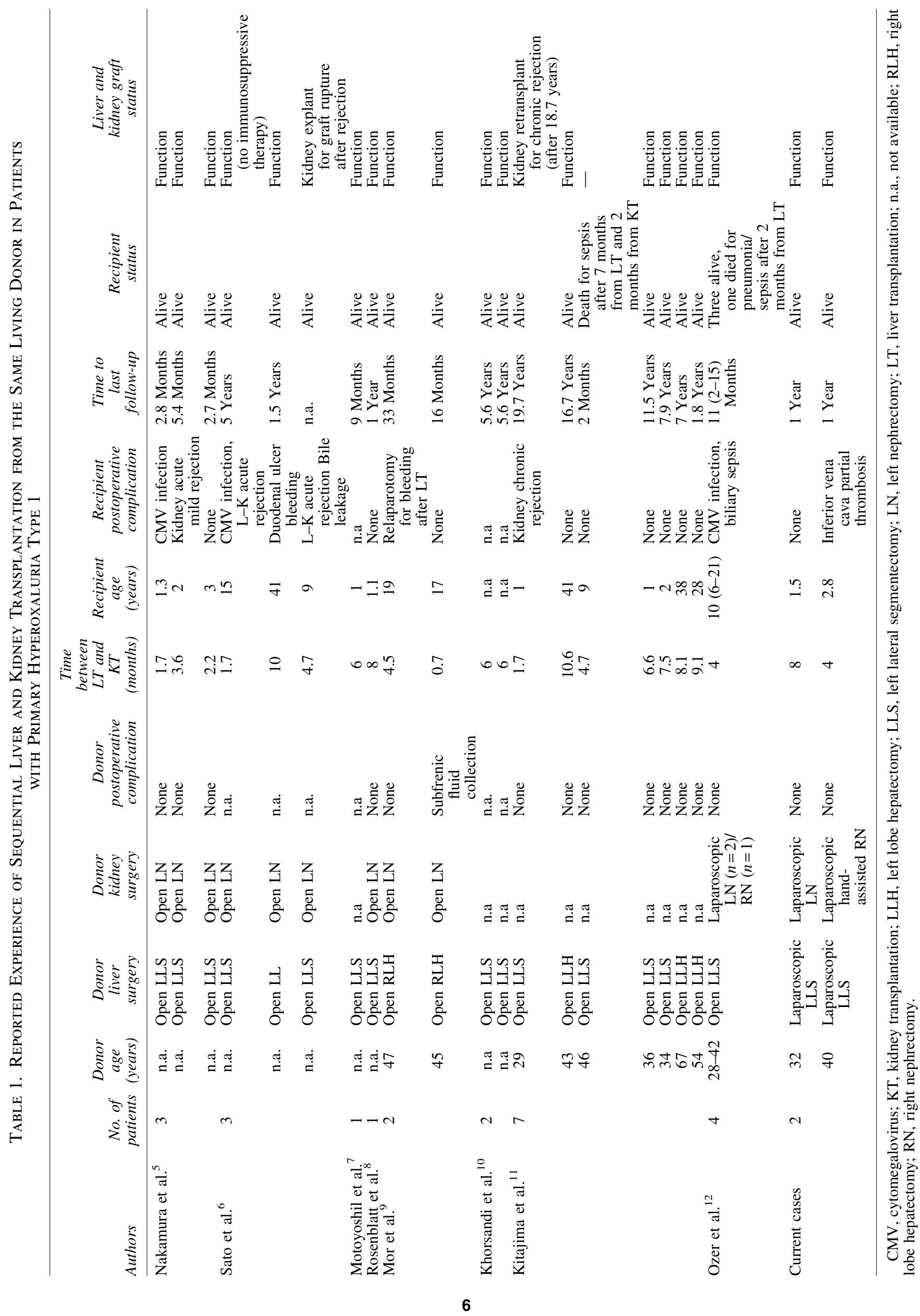




\section{Disclosure Statement}

No competing financial interests exist.

\section{Funding Information}

No funding was received for this article.

\section{References}

1. Cochat P, Rumsby G. Primary hyperoxaluria. N Engl J Med 2013;369:649-658.

2. Harambat J, Fargue S, Bacchetta J, Acquaviva C, Cochat P. Primary hyperoxaluria. Int J Nephrol 2011;2011:864580.

3. Bacchetta J, Mekahli D, Rivet C, Demède D, Leclerc AL. Pediatric combined liver-kidney transplantation: A 2015 update. Curr Opin Organ Transplant 2015;20:543-549.

4. Cochat P, Hulton SA, Acquaviva C, et al. Primary hyperoxaluria type 1: Indications for screening and guidance for diagnosis and treatment. Nephrol Dial Transplant 2012;27: $1729-1736$

5. Nakamura M, Fuchinoue S, Nakajima I, et al. Three cases of sequential liver-kidney transplantation from livingrelated donors. Nephrol Dial Transplant 2001;16:166-168.

6. Sato S, Fuchinoue S, Kimikawa M, et al. Sequential liverkidney transplantation from a living-related donor in primary hyperoxaluria type 1 (oxalosis). Transplant Proc 2003;35:373-374.

7. Motoyoshil Y, Hattori M, Chikamoto H, et al. [Sequential combined liver-kidney transplantation for a one-year-old boy with infantile primary hyperoxaluria type 1]. Nihon Jinzo Gakkai Shi 2006;48:22-28 (Article in Japanese).

8. Rosenblatt GS, Jenkins RD, Barry JM. Treatment of primary hyperoxaluria type 1 with sequential liver and kidney transplants from the same living donor. Urology 2006;68: 427.e7-427.e8.

9. Mor E, Nesher E, Ben-Ari Z, et al. Sequential liver and kidney transplantation from a single livingdonor in two young adults with primary hyperoxaluria type 1. Liver Transpl 2013;19:646-648.

10. Khorsandi SE, Samyn M, Hassan A, et al. An institutional experience of pre-emptive liver transplantation for pediatric primary hyperoxaluria type 1. Pediatr Transplant 2016;20: 523-529.

11. Kitajima K, Ogawa Y, Miki K, et al. Longterm renal allograft survival after sequential liver-kidney transplantation from a single living donor transplantation. Liver Transpl 2017;23:315-323.

12. Ozer A, Aktas H, Bulum B, Emiroglu R. The experience of combined and sequential liver and kidney transplantation from a single living donor in patients with primary hyperoxaluria type 1. Pediatr Transplant 2019;23:e13406.

13. Park JI, Kim KH, Lee SG. Laparoscopic living donor hepatectomy: A review of current status. J Hepatobiliary Pancreat Sci 2015;22:779-788.

14. Bekheit M, Khafagy PA, Bucur P, et al. Donor safety in live donor laparoscopic liver procurement: Systematic review and meta-analysis. Surg Endosc 2015;29:3047-3064.

15. Piros L, Langer RM. Laparoscopic donor nephrectomy techniques. Curr Opin Organ Transplant 2012;17:401-405.

16. Özdemir-van Brunschot DM, Koning GG, van Laarhoven $\mathrm{KC}$, et al. A comparison of technique modifications in laparoscopic donor nephrectomy: A systematic review and meta-analysis. PLoS One 2015;10:e0121131.

17. Soubrane O, Cherqui D, Scatton O, et al. Laparoscopic left lateral sectionectomy in living donors safety and repro- ducibility of the technique in a single center. Ann Surg 2006;244:815-820.

18. Rogiers X, Malagó M, Habib N, Broelsch CE. An easy technique for inferior vena cava control in pediatric liver transplantation. J Am Coll Surg 1996;182:555-556.

19. Ratner LE, Ciseck LJ, MooRE RG, Cigarroa FG, Kaufman HS, Kavoussi LR. Laparoscopic live donor nephrectomy. Transplantation 1995;60:1047-1049.

20. Wolf JS, Jr., Tchetgen MB, Merion RM. Hand-assisted laparoscopic live donor nephrectomy. Urology 1998;52: 885-887.

21. Calinescu AM, Wildhaber BE, Poncet A, Toso C, McLin VA. Outcomes of combined liver-kidney transplantation in children: Analysis of the scientific registry of transplant recipients. Am J Transplant 2014;14:2861-2868.

22. Cochat P, Fargue S, Harambat J. Primary hyperoxaluria type 1: Strategy for organ transplantation. Curr Opin Organ Transplant 2010;15:590-593.

23. Fonouni H, Mehrabi A, Golriz M, et al. Comparison of the laparoscopic versus open live donor nephrectomy: An overview of surgical complications and outcome. Langenbecks Arch Surg 2014;399:543-551.

24. Burg JM, Scott DL, Roayaie K, Maynard E, Barry JM, Enestvedt CK. Impact of center volume and the adoption of laparoscopic donor nephrectomy on outcomes in pediatric kidney transplantation. Pediatr Transplant 2018;22:e13121.

25. Abu Hilal M, Aldrighetti L, Dagher I, et al. The Southampton consensus guidelines for laparoscopic liver surgery: From indication to implementation. Ann Surg 2018;268: 11-18.

26. Han HS, Cho JY, Kaneko H, et al. Expert panel statement on laparoscopic living donor hepatectomy. Dig Surg 2018; 35:284-288.

27. Gautier S, Monakhov A, Miloserdov I, et al. Simultaneous laparoscopic left lateral sectionectomy and nephrectomy in the same living donor: The first case report. Am J Transplant 2019;19:1847-1851.

28. Villani V, Gupta N, Elias N, et al. Bilateral native nephrectomy reduces systemic oxalate level after combined liver-kidney transplant: A case report. Pediatr Transplant 2017;21:e12901.

29. Lee E, Ramos-Gonzalez G, Rodig N, Elisofon S, Vakili K, Kim HB. Bilateral native nephrectomy to reduce oxalate stores in children at the time of combined liver-kidney transplantation for primary hyperoxaluria type 1. Pediatr Nephrol 2018;33:881-887.

30. Au KP, Chok KSH. Minimally invasive donor hepatectomy, are we ready for prime time? World J Gastroenterol 2018;24:2698-2709.

31. Lentine KL, Lam NN, Segev DL. Risks of living kidney donation: current state of knowledge on outcomes important to donors. Clin J Am Soc Nephrol 2019;14:597-608.

Address correspondence to: Marco Spada, MD, PhD, FEBS Division of Abdominal Transplantation and Hepatobiliopancreatic Surgery Bambino Gesù Children's Hospital IRCCS Piazza Sant'Onofrio 4 Rome 00165 Italy

E-mail: marco.spada@opbg.net 